\title{
Measuring Drivers' Dynamic Seating Experience Using Pressure Mats
}

\author{
Songyi Chae, Gyouhyung Kyung*, and Kyunghyun Nam \\ School of Design \& Human Engineering, UNIST \\ 100 Banyeon-ri, Eonyang-eup, Ulju-gun, Ulsan, Korea \\ ghkyung@unist.ac.kr
}

\begin{abstract}
The objective of this study was to find the relationship between body-seat pressure distribution and driver comfort ratings of dynamic seating experience. A total of 38 participants performed four short-term driving sessions in a commercialized vehicle. These sessions involved two driving environments (lab vs. field-based). Body-seat interface pressure data were recorded continuously during driving, and the comfort ratings of the whole body and local body parts were measured after each session. Several body-seat pressure distribution variables were proposed to improve sitting comfort.
\end{abstract}

Keywords: Comfort, Driver Seat, Pressure Distribution.

\section{Introduction}

Driver seat is one of major factors which determine a driver's overall comfort and musculoskeletal health. With car customers' increasing concerns about safety and comfort, designing a proper driver seat has become a more challenging issue for car manufacturers. From an ergonomic perspective, comfort plays an important role in seat design (Juijt-Evers et al., 2003, Mehta and Tewari, 2000, Zhoa and Tang, 1994), as comfortable seats can reduce driver's back pain by alleviating vibration and road shock (Troup, 1978).

Many seat comfort measurement methods have been investigated. Objective measures include posture, the number of body movements, estimations of muscle activation and muscle fatigue by electromyography (EMG), pressure at the back rest and seat pan, model-estimations of spinal loading forces, stature loss, and foot volume changes. Subjective measures involve comfort and discomfort ratings on a whole body or local body parts.

As objective measurements, pressure variables often were used with subjective comfort ratings in some studies (e.g., de Looze et al., 2003; Andreoni et al., 2002). De Looze et al (2003) investigated comfort using both subjective evaluation and objective evaluation methods. Their study used a questionnaire for subjective evaluation and body pressure distribution variables on a massage chair as objective measures, which is based on the sitting comfort evaluation index model. Dhingra et al (2003) identified important factors for tractor seat discomfort by incorporating

\footnotetext{
* Corresponding author.
} 
quantitative and qualitative approaches (i.e., seat pressure distribution and seat comfort rating). In their study, however, the exclusive use of interface pressure did not successfully predicted car seat comfort. Similarly, Lee and Ferraiuolo (1993) concluded that there was no strong correlation between subjective comfort and body pressure distribution. Gyi and Porter (1999) showed that interface pressure and driving comfort had no clear relationship. More recent studies suggested to use different types of pressure variables such as total contact area, lateral load, lateral area, average pressure ratio, peak pressure ratio variables on different body parts (Kyung et al., 2008; Na et al., 2005; Andreoni et al., 2002). For preferred seatinterface pressure values, some studies suggested guidelines based on differences in gender, body mass index, stature, and seating position (e.g., Stinson, 2003). Kolich (2003) measured occupants' preferred contour and geometry characteristics of the seats and related these to anthropometric data.

The definitions of comfort and discomfort are not yet clearly defined and still on a debate (Helander \& Zang, 1997; Bishu et al., 1991). Several studies tried to conceptualize these two states (Hertzberg, 1958; Floyd and Robers, 1958) Zhang et al (1996) showed that comfort and discomfort had an orthogonal association. Kyung et al (2008) measured separate comfort and discomfort using derived versions of combinations of Borg (1990) and Corlett and Bishop (1976), and found that bodyseat interface pressure had a stronger relationship with overall and comfort ratings than with discomfort ratings which is in contrast to the model of de Looze (2003). To evaluate massage chair comfort, Yang et al (2009) designed Massage Chair Comfort Questionnaire based on 4 questionnaires about sitting comfort - General Comfort Rating (Shackel et al., 1969), Body Part Discomfort Rating (Corlet \& Biship, 1976), Overall Comfort Index (Kolich, 1999), and Automotive Seating Discomfort Questionnaire (Smith et al., 2006). To analyze the association of pressure data and comfort rating, different methods have been used, including statistical method ( $\mathrm{Na}$ et al., 2005; Kyung et al., 2008; Porter et al., 2003), and artificial neural network (Kolich et al., 2004).

The objective of this study was to find the relationship between body-seat pressure distribution and driver comfort ratings of dynamic seating experience.

\section{Methods}

\subsection{Overview of Experiment and Participants}

The experiment constituted four short-term driving sessions in a commercialized vehicle, Genesis 3300 (Hyundai Motor Company, Korea). Thirty-eight participants were recruited from the local community with following criteria - Drivers who had a driver's license for more than 3 years, normal or corrected-to-normal eye vision in both eyes, and no current musculoskeletal disorders, and aged between 35 and 65 . The participants were divided into three groups in accordance with their statures (Table 1). Perceived subjective comfort rating data and body-seat interface pressure data were obtained from a field-based (dynamic) and three lab-based (static) driving sessions. 
Table 1. Participant characteristics

\begin{tabular}{|c|c|c|c|}
\hline $\begin{array}{c}\text { Stature } \\
\text { group }\end{array}$ & $\begin{array}{c}\text { No. of participants } \\
\text { (male, female) }\end{array}$ & $\begin{array}{c}\text { Mean (SD) } \\
\text { stature (cm) }\end{array}$ & $\begin{array}{c}\text { Mean (SD) mass } \\
(\mathbf{k g})\end{array}$ \\
\hline $\begin{array}{c}\text { Short } \\
\left(<\mathbf{1 6 5} \mathbf{c m}^{\mathbf{a}}\right)\end{array}$ & $12(2,10)$ & $155.6(5.0)$ & $61.9(9.1)$ \\
\hline Middle & $16(13,3)$ & $165.3(4.5)$ & $65.6(7.6)$ \\
\hline $\begin{array}{c}\text { Tall } \\
\left(>\mathbf{1 7 5} \mathbf{c m}^{\mathbf{b}}\right)\end{array}$ & $10(10,0)$ & $179.0(3.4)$ & $85.3(8.5)$ \\
\hline Total & $38(25,13)$ & $165.7(9.9)$ & $69.3(12.5)$ \\
\hline
\end{tabular}

Source: NHANES III (1994).

a18th or lower percentiles of gender-mixed population.

b90th or higher percentiles of gender-mixed population.

\subsection{Variables}

In the current study, stature group and driving venue were considered as independent variables. Body-interface pressure was recorded during driving sessions and subjective comfort ratings were surveyed after each session. Pressure data were collected from bilateral buttocks and backs. A whole body comfort rating, left/right shoulders and bilateral buttocks comfort ratings were used as subjective measurement. The rating scales ranged from 0 (Not at all comfortable) to 100 (Extremely comfortable).

\subsection{Experimental Protocols}

Two pressure mats were placed on both seat back and seat cushion and participants were seated carefully. At the first lab-based session, the instructor adjusted the steering wheel and seat, while, at the second and third lab-based sessions, participants adjusted the steering wheel and seat themselves, except the seat back angle was fixed at $25^{\circ}$ at the time.

\subsection{Data Collection Procedures and Processing}

Interface pressure data were recorded by two Tekscan (South Boston, MA, USA) pressure mats (5330 CONFORMatTM). Each pressure mat consists of $1024(32 \times 32)$ thin $(1.78 \mathrm{~mm})$ resistive sensors that could easily conform to the contour of the seat, and measure up to $255 \mathrm{mmHg}$ (5 PSI). Each mat had an active area of $471.4 \mathrm{~mm} \times$ $471.4 \mathrm{~mm}$, and sensor pitch was $14.73 \mathrm{~mm}(0.5$ sensor $/ \mathrm{cm} 2)$. Pressure data were divided into four parts (Figure 1).

\subsection{Data Analysis}

To analyze pressure data, 27 pressure variables were made (Table 2), which were related to average contact areas and ratio, average contact pressure and ratios, and average peak contact pressure and ratio. ANOVA and MANOVA $(\alpha=0.05)$ were used and the associations between pressure variables were evaluated by principal 


\begin{tabular}{|c|c|}
\hline $\begin{array}{c}\text { Left Back } \\
(32 \times 16)\end{array}$ & $\begin{array}{c}\text { Right Back } \\
(32 \times 16)\end{array}$ \\
& \\
\hline $\begin{array}{c}\text { Left Buttock } \\
(32 \times 16)\end{array}$ & $\begin{array}{c}\text { Right Buttock } \\
(32 \times 16)\end{array}$ \\
& \\
\hline
\end{tabular}

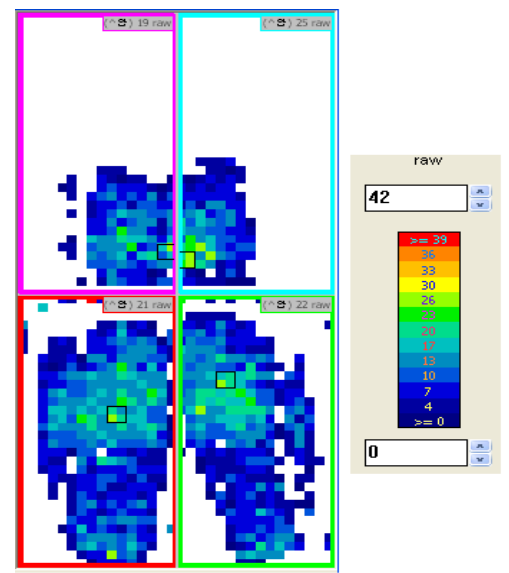

Fig. 1. Two pressure mats for four local body parts (left: number of sensors in parentheses, right: pressure distribution)

component analysis (PCA). Regression of subjective comfort rating on the factors from the PCA was used to determine a linear combination of the factors that can predict driver sitting comfort. Factors were selected by the eigenvalue $(>1)$ and the cumulative percentage $(\$ 90 \%)$ of variance accounted for by the selected factors (Lehman et al., 2005). The resulting factors were rotated by the varimax method.

Table 2. Pressure variables

\begin{tabular}{|c|c|c|}
\hline Group & Variable name & Description \\
\hline \multirow{3}{*}{ Average contact area $\left(\mathrm{cm}^{2}\right)$} & aBTL (aBTR) & Left (right) buttock \\
\hline & aLB (aRB) & Left (right) back \\
\hline & aSUM & $\mathrm{aBTL}+\mathrm{aBTR}+\mathrm{aLB}+\mathrm{aRB}$ \\
\hline Average contact area ratio & \multicolumn{2}{|c|}{ aBTL/aSUM, aBTR/aSUM, aLB/aSUM, aRB/aSUM } \\
\hline \multirow{3}{*}{ 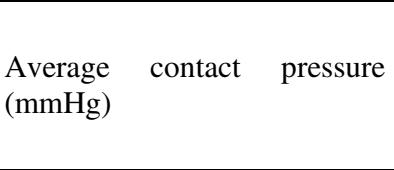 } & avgBTL (avgBTR) & Left (right) buttock \\
\hline & avgLB (avgRB) & Left (right) back \\
\hline & $\operatorname{avgSUM}$ & $\begin{array}{l}\text { avgBTL + avgBTR + avgLB } \\
+ \text { avgRB }\end{array}$ \\
\hline Average contact pressure ratio & \multicolumn{2}{|c|}{$\begin{array}{l}\text { avgBTL/avgSUM, avgBTR/avgSUM, avgLB/avgSUM, } \\
\text { avgRB/avgSUM }\end{array}$} \\
\hline \multirow{3}{*}{$\begin{array}{l}\text { Average peak contact } \\
\text { pressure }(\mathrm{mmHg})\end{array}$} & pkBTL (pkBTR) & Left (right) buttock \\
\hline & pkLB (pkRB) & Left (right) back \\
\hline & pkSUM & $\begin{array}{l}\text { pkBTL + pkBTR + pkLB }+ \\
\text { pkRB }\end{array}$ \\
\hline $\begin{array}{l}\text { Average peak contact } \\
\text { pressure ratio }\end{array}$ & \multicolumn{2}{|c|}{$\begin{array}{l}\text { pkBTL/pkSUM, } \\
\text { pkRB/pkSUM }\end{array}$} \\
\hline
\end{tabular}




\section{Results}

\subsection{Effects of Stature and Driving Venue on Interface Pressures}

MANOVA showed a significant $(\mathrm{p} \leq 0.0001)$ main effect of Stature and Driving Venue on the 27 pressure variables. There was no significant interaction effect of Stature $\times$ Driving Venue. From subsequent ANOVAs, stature effects were found on average contact areas, average pressure, maximum peak pressure and ratios (aRB, aLB, aBTR, aBTL, aSUM, aLB/aSUM, aBTL/aSUM, avgLB, avgLB/avgSUM, pkRB and $\mathrm{pkBRT} / \mathrm{pkSUM}$ ) with $\mathrm{p} \leq 0.03$. Driving Venue effect was found only on peak contact pressure ratio (pkBTL/pkSUM). Significant mean differences between lab and field-based sessions were found for pkBTL $(\mathrm{p} \leq 0.024)$, and pkBTL/pkSUM $(\mathrm{p} \leq$ 0.007), and subject comfort rating variables for the left back and bilateral buttocks. All subject comfort ratings were higher in field-based than lab-based sessions. Significant difference of Driving Venue effects was found on the peak pressure variable at the left buttock. On lab-based sessions, the mean (SD) of the peak pressure ratio at the left buttock was $0.270(0.084)$, which was lower than that on field-based venue, 0.335 (0.359).

\subsection{Correlations between Subjective Comfort Ratings and Pressure Variables}

Whole body and local body parts comfort ratings were higher on field-based than labbased sessions. Comparing bilateral means of subjective comfort ratings, the left back and buttock were higher than the right. Mean average contact area was higher on the right parts. Among the whole contact area variables, right buttock ratio was the highest. As for average pressure variables, the right back and buttock were higher than the left ones as well. Right buttock ratio (avgBTR/avgSUM) was the highest. Peak pressure variables at the right back and buttock were higher than the left. $\mathrm{pkBTR} / \mathrm{pkSUM}$ value was the highest one.

\subsection{PCA and Regression Analysis Using Pressure Variables}

With an eigenvalue $>1$, nine factors were accounted for $89.0 \%$ of the total variance (Table 3). Based on the smallest communality of 0.739 (aBTL/aSUM), the variance of each pressure variable was well accounted for by the selected factors. For Factors 1 and 4 related to contact area ratios, coefficients with opposite signs were found between the left buttock and bilateral back (i.e., aBTL/aSUM vs. a(L or R)B/aSUM), and between the back and right buttock pressure ratios (i.e., pkBTR, pkSUM vs. pk(R or L)B/pkSUM), indicating negative associations between the bilateral back and each of buttocks. For Factor 2, coefficients relating to pressure of bilateral backs and buttocks were all positive.

Regression analyses showed adjusted $\mathrm{R} 2=-0.033$ between the subjective comfort ratings and the nine factors. Fitted models for comfort ratings were not significant. Based on the size of the standard beta weights (Table 4), increasing Factor 6 and Factor 7 would be effective at improving whole body comfort rating. The largest and positive beta weight for Factor 6 indicated that increasing pressure at the right buttock 
would be the most effective method for improving whole body comfort rating. Similarly, the second largest beta weight for Factor 7 suggested that increasing pressure at left back would be the second most effective way of improving the whole body comfort rating. Factor 4 had the third largest weights, suggesting the third strategy of increasing contact area at the buttocks.

Table 3. Nine principal components after Kaiser normalized varimax rotation (underlined values are $>0.6$ )

\begin{tabular}{|c|c|c|c|c|c|c|c|c|c|}
\hline & Factor 1 & Factor 2 & Factor 3 & Factor 4 & Factor 5 & Factor 6 & Factor 7 & Factor 8 & Factor 9 \\
\hline $\begin{array}{l}\text { Pressure } \\
\text { variable }\end{array}$ & $\begin{array}{c}\text { Back vs. } \\
\text { Left } \\
\text { buttock } \\
\text { (area) }\end{array}$ & $\begin{array}{c}\text { Back \& } \\
\text { buttock } \\
\text { (pressure) }\end{array}$ & $\begin{array}{c}\text { Back vs. } \\
\text { Right } \\
\text { buttock } \\
\text { (pressure) }\end{array}$ & $\begin{array}{c}\text { Buttock } \\
\text { (area) }\end{array}$ & $\begin{array}{c}\text { Back \& } \\
\text { Left } \\
\text { buttock } \\
\text { (pressure) }\end{array}$ & $\begin{array}{c}\text { Right } \\
\text { buttock } \\
\text { (pressure) }\end{array}$ & $\begin{array}{l}\text { Left back } \\
\text { (pressure) }\end{array}$ & $\begin{array}{l}\text { Right back } \\
\text { (pressure) }\end{array}$ & $\begin{array}{c}\text { Left } \\
\text { buttock } \\
\text { (pressure) }\end{array}$ \\
\hline aBTR/aSUM & -.861 & -.112 & .163 & .169 & .061 & .105 & .129 & -.073 & .065 \\
\hline aLB & .857 & -.002 & .102 & .120 & .122 & .157 & .269 & -.169 & -.025 \\
\hline aBTL/aSUM & $-\overline{-.764}$ & .087 & .086 & .103 & .198 & .250 & .133 & -.092 & .038 \\
\hline aLB/aSUM & .754 & -.004 & .102 & -.350 & .141 & .163 & .304 & -.215 & -.013 \\
\hline $\mathrm{aRB}$ &.$\overline{701}$ & .046 & .196 & .336 & .127 & .427 & -.039 & .205 & .077 \\
\hline aRB/aSUM & $\overline{.561}$ & .064 & .212 & -.310 & .184 & .528 & -.052 & .226 & .088 \\
\hline avgBTR & -.022 & .965 & .005 & -.003 & -.130 & .125 & -.057 & -.064 & -.060 \\
\hline avgLB & -.077 & .860 & -.003 & -.051 & -.089 & -.090 & .371 & .197 & -.102 \\
\hline $\operatorname{avgBTL}$ & .139 & .859 & -.071 & .100 & .070 & .026 & .047 & -.058 & .235 \\
\hline avgRB & .063 & .766 & .021 & -.119 & .013 & -.107 & .179 & .486 & -.174 \\
\hline $\begin{array}{c}\text { avgBTR/avgS } \\
\text { UM }\end{array}$ & -.073 & .629 & .114 & .038 & .459 & .383 & -.146 & -.277 & .043 \\
\hline pkSUM & .055 & .046 & -.854 & .025 & -.243 & .253 & .120 & .223 & .248 \\
\hline pkRB/pkSUM & .034 & -.025 & .810 & .099 & .030 & -.036 & .032 & .448 & .233 \\
\hline $\mathrm{pkLB} / \mathrm{pkSUM}$ & .118 & .094 & .731 & .027 & .030 & -.124 & .556 & .104 & .099 \\
\hline aBTL & -.177 & .070 & .032 & .887 & .115 & .137 & .042 & -.023 & -.003 \\
\hline aBTR & -.332 & -.083 & .119 & .876 & .010 & .025 & .045 & -.007 & .035 \\
\hline aSUM & .418 & .003 & -.102 & .838 & -.129 & -.205 & -.135 & .080 & -.047 \\
\hline avgSUM & -.067 & -.117 & -.010 & -.105 & -.902 & .157 & .038 & -.054 & -.041 \\
\hline $\begin{array}{c}\text { avgBTL/avgS } \\
\text { UM }\end{array}$ & .080 & -.171 & .085 & .080 & .733 & .185 & -.009 & -.277 & .382 \\
\hline $\begin{array}{c}\operatorname{avgRB} / \operatorname{avgSU} \\
M\end{array}$ & .011 & -.186 & .225 & -.210 & .708 & .006 & .182 & .462 & -.158 \\
\hline $\begin{array}{c}\operatorname{avgLB} / \operatorname{avgSU} \\
M\end{array}$ & -.195 & .023 & .171 & -.105 & .632 & -.062 & .601 & .147 & -.064 \\
\hline $\begin{array}{c}\mathrm{pkBTR} / \mathrm{pkSU} \\
\mathrm{M}\end{array}$ & -.004 & .058 & -.248 & .043 & -.058 & .869 & -.096 & -.130 & -.083 \\
\hline pkBTR & .044 & .023 & -.612 & .015 & -.132 & .717 & .011 & .017 & .042 \\
\hline pkLB & .107 & .234 & $\overline{-.030}$ & .024 & .006 & -.066 & .861 & .262 & .066 \\
\hline pkRB & .005 & .106 & .091 & .072 & .057 & -.042 & .271 & .865 & .138 \\
\hline pkBTL & -.075 & .028 & -.232 & -.060 & .117 & -.046 & .077 & .073 & .905 \\
\hline $\begin{array}{c}\mathrm{pkBTL} / \mathrm{pkSU} \\
\mathrm{M}\end{array}$ & -.007 & -.029 & .496 & .056 & -.009 & .004 & -.029 & .067 & .816 \\
\hline Eigenvalue & 3.871 & 3.589 & 2.935 & 2.768 & 2.747 & 2.250 & 1.972 & 1.969 & 1.944 \\
\hline Cum percent & 14.336 & 27.630 & 38.501 & 48.753 & 58.926 & 67.258 & 74.564 & 81.855 & 89.056 \\
\hline
\end{tabular}


Table 4. Regression coefficients and standard beta weights for regression models relating PCA factors to whole body comfort ratings

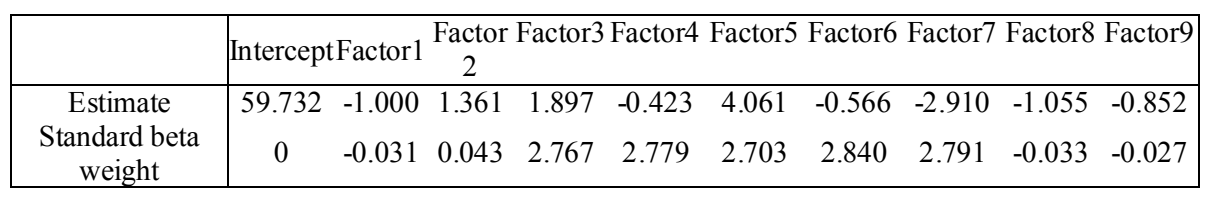

\section{Discussion}

The current study focused on sitting comfort during short-term driving, and used interface pressure data as objective measures with subjective comfort rating responses. Most average pressure variables showed a difference between lab-based and field-based drivings, as field-based driving showed higher comfort rating for whole body and local body parts and comfort ratings were higher at the left back and buttocks than right parts.

Different stature groups had significantly different values of all average contact area $(\mathrm{p}=0.000)$ and ratio $(\mathrm{p}<0.016)$, average contact pressure in their left and right back $(\mathrm{p}<0.025)$, average pressure ratio in their left buttock $(\mathrm{p}=0.016)$ and peak pressure in their right back $(\mathrm{p}=0.000)$. From a PCA, contrasting associations were found between local body parts in terms of contact area and contact pressure: negative associations between the left buttock and bilateral back from contact area and between the right buttock and bilateral back from average pressure. Driving appears to require asymmetrical body postures based on bilaterally different pressure values. The regression results suggested that increasing pressure at the right buttock would be the most effective method for improving whole body comfort rating and increasing pressure at left back would be the second most effective way of improving the whole body comfort rating.

\section{Conclusions}

Some pressure variables on average contact area, average and peak contact pressure ratio could be used for the assessment of driver sitting comfort. Driving experience that requires asymmetric postures could be improved by providing balanced pressure between bilateral body parts for ergonomic human-seat interface. For future study, a more comprehensive experiment is needed to include factors such as BMI, seat angles, and seating position.

\section{References}

1. Lehman, N., O'Rourke, L., Hatcher, E.J.: JMP for Basic Univariate and Multivariate Statistics: A Step-by-Step Guide. SAS Institue Inc., Cary (2005)

2. Shackel, K.D., Chidsey, P.: The assessment of chair comfort. Ergonomics 12, 269-306 (1969) 
3. Metha, C.R., Tewari, V.R.: Seating discomfort for tractor operators - a critical review. International Journal of Industrial Ergonomics 25, 661-674 (2000)

4. De Looze, M.P., De Kuijt-Evers, L.F.M., Van Dieen, J.: Sitting comfort and discomfort and the relationships with objective measures. Ergonomics 46(10), 985-997 (2003)

5. Gyi, D.E., Porter, J.M.: Interface pressure and the prediction of car seat discomfort. Applied Ergonomics 30, 99-107 (1998)

6. Smith, D.R., Andrews, D.M., Wawrow, P.T.: Development and evaluation of the Automotive Seating Discomfort Questionnaire (ASDQ). International Journal of Industrial Ergonomics 36, 141-149 (2006)

7. Corlett, E.N., Bishop, R.P.: A technique for assessing postural discomfort. Ergonomics 19, 175-182 (1976)

8. Andreoni, G., Santambrogio, G.C., Rabuffetti, M., Pedotti, A.: Method for the analysis of posture and interface pressure of car drivers. Applied Ergonomics 33, 511-522 (2002)

9. Kyung, G., Nussbaum, M.A.: Driver sitting comfort and discomfort (part2): Relationships with and prediction from interface pressure. International Journal of industrial Ergonomics 38, 526-538 (2008)

10. Dhingra, H.S., Tewari, V.K., Singh, S.: Discomfort, Pressure Distribution and Safety in Operator's Seat - A Critical Review, Agricultural Engineering international: the CICG Journal of Scientific Research and Development. Invited Overview Paper 5 (2003)

11. Hertzberg, H.T.E.: Seat comfort. In: Hansen, R., Cnrnog, D.R., Hertzberg, H.T.E. (eds) Annotated Bibliography of Applied Physical Anthropology in Human Engineering, WADC Technical report 56-30, 297-300 (1958)

12. Lee, J., Ferraiuolo, P.: Seat comfort. SAE Technical Paper no. 930105 (1993)

13. Kuijt-Evers, L.F.M., Krause, F., Vink, P.: Aspects to improve cabin comfort of wheel loaders and excavators according to operators. Applied Ergonomics 34, 265-271 (2003)

14. Stinson, M.D., Armstrong, A.P., Eakin, P.: Seat-Interface Pressure: A Pilot Study of the Relationship to Gender, Body Mass Index, and Seating Position. Arch. Phys. Med. Rehabil. 84 (2003)

15. Helander, M.G., Zhang, L.: Field studies of comfort and discomfort in sitting. Ergonomics 40, 895-915 (1997)

16. Kolich, M.: Reliability and Validity of an Automobile Seat Comfort Survey, Technical Paper No. 1999-01-3232 (1999)

17. Kolich, M.: Automobile seat comfort: occupant preferences vs. anthropometric accommodation. Applied Ergonomics 34, 177-184 (2003)

18. Bishu, R.R., Hallbeck, M.S., Riley, M.W.R.: Seating comfort and its relationship to spinal profile: a pilot study. International Journal of Industrial Ergonomics 8, 89-101 (1991)

19. Na, S., Lim, S., Choi, H.S., Chung, M.K.: Evaluation of driver's discomfort and postural change using dynamic body pressure distribution. International Journal of Industrial Ergonomics 35, 1096-1985 (2005)

20. Troup, J.D.G.: Driver's back pain its prevention: A review of the postural, vibratory and muscular factors, together with the problem of transmitted road-shock. Applied Ergonomics 9(4), 207-214 (1978)

21. Floyd, W.F., Roberts, D.F.: Anatomical and physiological principles in chair and table design. Ergonomics 2, 1-16 (1958)

22. Zhang, L., Helander, M., Drury, C.: Identifying factors of comfort and discomfort. Human Factors 38(3), 377-389 (1996)

23. Zhoa, J.H., Tang, L.: An evaluation of comfort of a bus seat. Applied Ergonomics 25, 386392 (1994) 